\title{
Marangoni bursting: Evaporation-induced emulsification of a two-component droplet
}

\author{
Guillaume Durey, ${ }^{1, *}$ Hoon Kwon, ${ }^{1}$ Quentin Magdelaine, ${ }^{1}$ Mathias Casiulis, ${ }^{1}$ Julien Mazet, ${ }^{1,2}$ \\ Ludovic Keiser, ${ }^{3, \dagger}$ Hadrien Bense,${ }^{3}$ Pierre Colinet, ${ }^{4}$ José Bico, ${ }^{3}$ and Etienne Reyssat ${ }^{3}$ \\ ${ }^{1}$ The Lutetium Project, ESPCI Paris, PSL Research University, Paris, France \\ ${ }^{2}$ Conservatoire National Supérieur de Musique et de Danse de la Ville de Paris, \\ PSL Research University, Paris, France \\ ${ }^{3}$ UMR CNRS 7636 PMMH, ESPCI Paris, PSL Research University, Sorbonne Universités, \\ Sorbonne Paris Cité, Paris, France \\ ${ }^{4}$ TIPS Laboratory, Université Libre de Bruxelles, Brussels, Belgium
}

(Received 2 February 2018; published 17 October 2018)

\begin{abstract}
This paper is associated with a video winner of a 2017 APS/DFD Milton van Dyke Award for work presented at the DFD Gallery of Fluid Motion. The original video is available from the Gallery of Fluid Motion, https://doi.org/10.1103/APS.DFD.2017.GFM.V0020
\end{abstract}

DOI: 10.1103/PhysRevFluids.3.100501

A drop of water deposited on the surface of a bath of oil forms a quiescent floating lens. Conversely, a drop of pure isopropanol on the surface of the oil spontaneously spreads. In this video, we describe the spectacular phenomenon occurring as a binary drop of alcohol and water is deposited on a bath of sunflower oil. The drop spontaneously spreads but also fragments into a myriad of tiny droplets [1]. Increasing the initial fraction of alcohol in the mixture strongly reduces the size of the ejected droplets (Fig. 1).

This phenomenon belongs to a wider class of instabilities involving surface tension gradients induced by evaporation or diffusion of one component of a binary fluid. These include the spreading of soluble surfactants [2], self-pulsating drops [3,4], and the well-known example of tears of wine $[5,6]$.

Above a threshold alcohol concentration, the binary mixture wets the oil, hence the droplet spreads. The volatile alcohol evaporates, and the thinner regions at the periphery of the drop become richer in water. The local concentration at the periphery becomes smaller than at the threshold, and the spreading stops (Fig. 2). The spatially inhomogeneous mixture concentration yields surface tension gradients along the radius of the drop. They drive solutal Marangoni flows from the center of the drop outward, which are opposed by viscous flows in the supporting oil layer. Combining these physical ingredients enables us to predict the maximum spreading radius of the drop and the time at which it retracts [1]. The antagonistic outward Marangoni flow and dewetting phenomenon at the periphery result in the mixture accumulating in a rim at the edge of the drop. This rim is unstable and breaks up into a myriad of satellite droplets. The detailed mechanisms for the formation of droplets and their transport away from the mother drop (Fig. 3) remain open questions.

\footnotetext{
*guillaume.durey@espci.org
}

†ludovic.keiser@espci.org

Published by the American Physical Society under the terms of the Creative Commons Attribution 4.0 International license. Further distribution of this work must maintain attribution to the author(s) and the published article's title, journal citation, and DOI. 

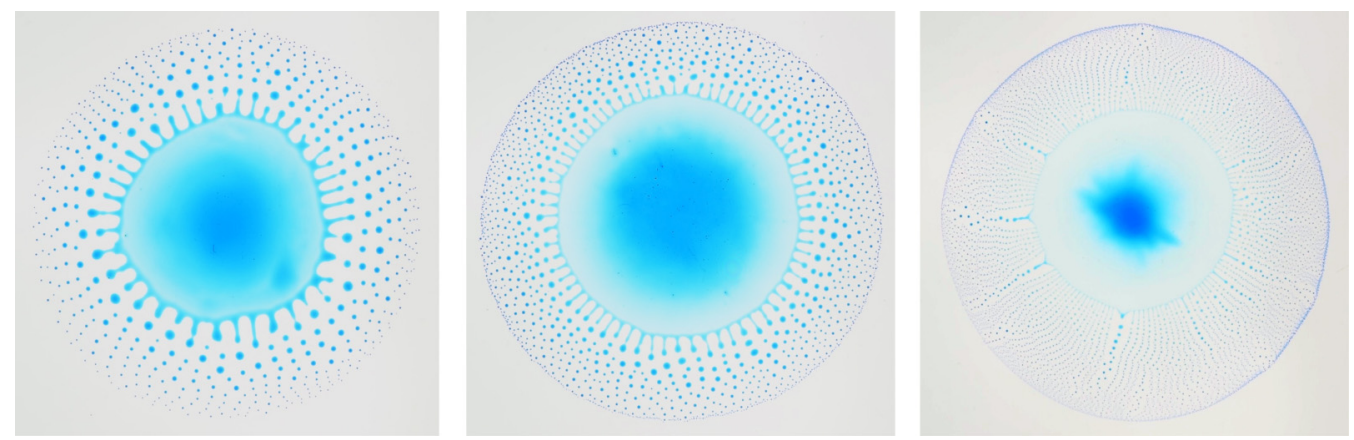

FIG. 1. The mass fraction of alcohol is increased from 0.4 (left) to 0.5 (right), which decreases the size of the ejected droplets. The central drop is about $4 \mathrm{~cm}$ in diameter. DOI: https://doi.org/10.1103/ APS.DFD.2017.GFM.V0020

This experiment caught the eye of a group of Ph.D. students in physics based in Paris, France, running a science YouTube channel called The Lutetium Project [7]. It aims at giving the general public an accurate and engaging perception of the work that scientists conduct in their labs. Visually striking present-day research experiments are featured on the channel and explained in an accessible yet precise way. Special attention is given to making the videos aesthetically pleasing and professional looking by fostering collaborations with students in graphic design and musical composition and working under the supervision of a student in computer graphics.

In the case of the Marangoni bursting experiment, the three key ingredients of the instability are visually introduced in the first part of the scenario: water doesn't wet the oil, ethanol wets the oil, and ethanol evaporates. The second part of the video reveals the Marangoni bursting in all its splendor. The third part consists of slow-motion footage, allowing for a more detailed explanation of the physical processes at play, leading up to the formation of the satellite droplets.

The soundtrack was composed to illustrate both the visuals and the physical phenomenon. Throughout, the soft timbre of several background instruments evokes the fluid-like nature of the interacting liquids. The crystalline order of the satellite droplets is reflected in a high-pitched melody, whose tempo varies accordingly with the speed of the footage. In addition, several overlaying musical phrases with different tonalities and dynamics give the impression that some notes fade away from the listener: this represents the ejection of the satellite droplets from the mother drop.

This video is part of a larger series found on The Lutetium Project's YouTube channel, called "Experiments in Music," that all follow the same creative procedure.
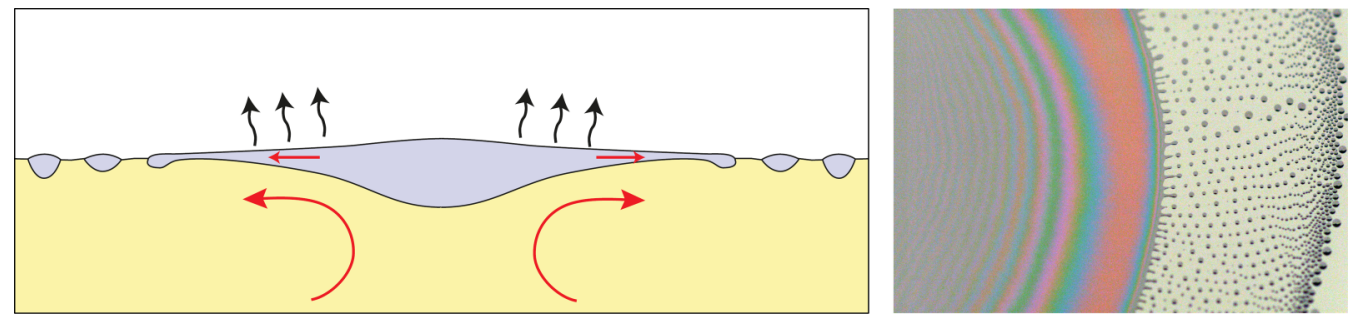

FIG. 2. Left: Side sketch of a spreading drop, representing the evaporative flux (dark arrows) and the radial decrease of the thickness, confirmed by interferometry. The Marangoni stress in the drop is balanced by the viscous stress in the oil bath, leading to a bulk flow (red arrows). Right: White light interferometry enables us to determine the local thickness $h$ of the spreading drop. DOI: https://doi.org/10.1103/APS.DFD.2017.GFM.V0020 


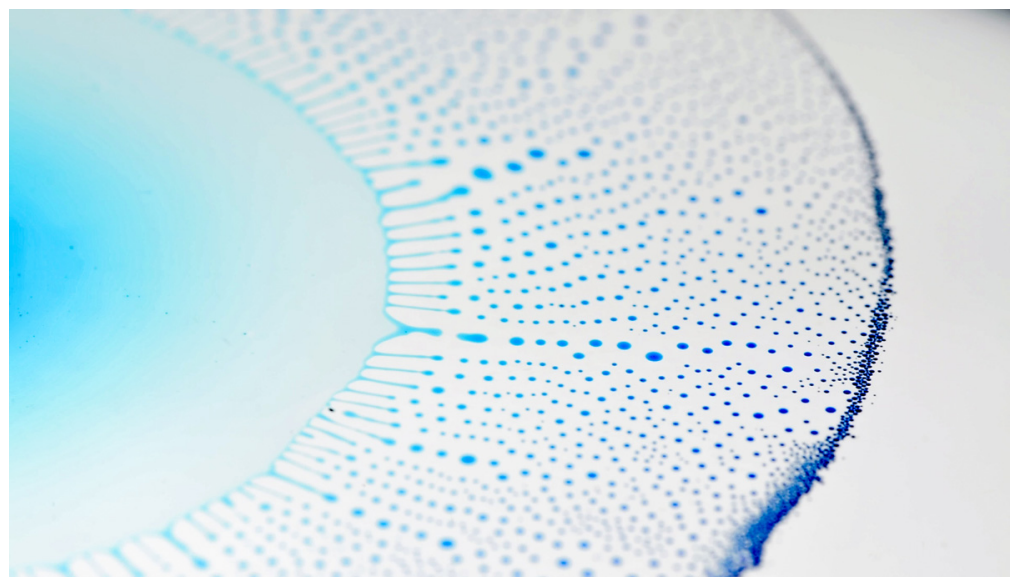

FIG. 3. Closeup of the unstable rim at the periphery of the mother drop. The physical mechanism underlying the destabilization of the rim remains an open question. DOI: https://doi.org/10.1103/ APS.DFD.2017.GFM.V0020

The present study was stimulated by an experiment often performed by Professor Gustavo Gutiérrez, at Simón Bolívar University in Caracas, Venezuela, as part of his numerous outreach activities aimed at school children. L.K. is grateful to Total S.A. for financial support. P.C. acknowledges financial support of the Fonds de la Recherche Scientifique (FNRS). The Lutetium Project acknowledges financial support from Espace des sciences Pierre-Gilles de Gennes, PSL Research University (IDEX ANR-10-IDEX-0001-02 PSL), ESPCI Paris, ESPCI Alumni, and Fonds ESPCI Paris.

[1] L. Keiser, H. Bense, P. Colinet, J. Bico, and É. Reyssat, Marangoni Bursting: Evaporation-Induced Emulsification of Binary Mixtures on a Liquid Layer, Phys. Rev. Lett. 118, 074504 (2017).

[2] M. Roché, Z. Li, I. Griffiths, S. Leroux, I. Cantat, A. Saint-Jalmes, and H. A. Stone, Marangoni Flow of Soluble Amphiphiles, Phys. Rev. Lett. 112, 208302 (2014).

[3] C. Antoine and V. Pimienta, Mass-spring model of a self-pulsating drop, Langmuir 29, 14935 (2013).

[4] F. Wodlei, J. Sebilleau, J. Magnaudet, and V. Pimienta, Marangoni-driven flower-like patterning of an evaporating drop spreading on a liquid substrate, Nat. Commun. 9, 820 (2018).

[5] J. B. Fournier and A. M. Cazabat, Tears of wine, Europhys. Lett. 20, 517 (1992).

[6] A. E. Hosoi and J. W. Bush, Evaporative instabilities in climbing films, J. Fluid Mech. 442, 217 (2001).

[7] The Lutetium Project, https://youtube.com/thelutetiumproject 